\title{
LEED Observation of Methane Monolayer Physisorbed on Ag(111)
}

\author{
Makoto SAKURAI, Fumiharu NAKAJIMA, Masato YASUDA and Takao NANBA \\ Department of Physics, Kobe University, Rokko-dai, Nada, Kobe 657-8501, Japan
}

(Received December 4, 2002, Accepted February 1, 2003)

\begin{abstract}
The structure of $\mathrm{CH}_{4}$ monolayer physisorbed on a $\mathrm{Ag}(111)$ surface was observed by LEED. The LEED patterns were recorded with suppressed electron current using a cooled CCD camera in order to minimize the electron stimulated desorption of adsorbed $\mathrm{CH}_{4}$. The LEED pattern indicated that the lattice of $\mathrm{CH}_{4}$ molecules forms hexagonal structure in rotationally ordered incommensurate state. Under specific equilibrium condition, intermediate state with the coverage less than unity was found to exist.
\end{abstract}

\section{Introduction}

The microscopic structure of the crystalline monolayer of molecules physisorbed on graphite have been studied for various simple molecules by low-energy electron diffraction (LEED). ${ }^{1-3)}$ For metal substrates, especially rare gas layers have been studied. ${ }^{1,4,5)}$ Among metal substrates, $\operatorname{Ag}(111)$ surface has been often used for physisorption systems due to its inert nature. On $\mathrm{Ag}(111)$ substrate, rare gas atoms adsorb in layer-by-layer manner, which means that the isotherm curve shows a sharp riser from almost zero coverage to full coverage at specific pressure. The rare gas monolayer is known to form a close packed hexagonal structure in azimuthally commensurate relation with the substrate lattice.

The vibrational excitation processes of several kinds of physisorbed molecules have been investigated by electron impact. ${ }^{6-10)}$ Generally, excitation cross section is affected by the structure of adsorbed molecules, and the adsorption scheme strongly depends on the characteristics of individual molecule; polar molecules such as carbon dioxide are not likely to show layer-by-layer growth. ${ }^{8}$ On the other hand, $\mathrm{CH}_{4}$ molecules forms well ordered monolayer. ${ }^{9,11,12)}$ We have measured that the incident energy dependence of elastic or vibrational excitation cross-section (I-V curve) is modulated by diffraction effect that arises from the generation of surface wave diffracted by substrate or adsorbate lattice. ${ }^{11,12)}$ In that experiments, LEED observation of $\mathrm{CH}_{4}$ layer was insufficient and the analysis of vibrational excitation process on the structural viewpoint remains incomplete.

In the present paper, we present the results of
LEED observation of $\mathrm{CH}_{4}$ layer physisorbed on a $\mathrm{Ag}(111)$ surface at various adsorption equilibrium conditions, and the growth mode specific to $\mathrm{CH}_{4} /$ $\mathrm{Ag}(111)$ system is discussed.

\section{Experimental}

The experimental apparatus is based on an ultrahigh vacuum chamber evacuated by a titanium sublimation pump with a liquid nitrogen shroud, a sputter-ion pump $(230 \mathrm{l} / \mathrm{s})$ and a turbo molecular pump $(450 \mathrm{l} / \mathrm{s})$. The chamber comprises a sample manipulating system with temperature control, a sputter-ion gun, a LEED optics (Omicron, SPECTALEED) and a high-resolution electron-energyloss spectrometer (HREELS; LK Technologies, ELS5000). The schematic drawing of the apparatus is shown in Fig. 1. Two-fold magnetic shields are installed in the vacuum chamber; outer one covers the LEED and EELS stages and inner one covers only the EELS stage. The sample manipulating system consists of XYZ-translator (Vacuum Generators), a differentially pumped rotational platform (Thermionics, RNN250) and a cryogenic closed-cycle refrigerator (IWATANI, Cryo Mini). The rotational platform is evacuated by a small turbomolecular pump $(70 \mathrm{l} / \mathrm{s})$ and a scroll pump. The substrate is mounted on a sample holder that is connected to the cold end of the refrigerator via a high-purity $(6 \mathrm{~N})$ copper rod and insulating sapphire crystal plates. A resistive heater and $\mathrm{Au}-0.07$ $\% \mathrm{Fe}$ vs. Chromel thermocouple are attached on the sample holder for the temperature control. The system enables XYZ-translation, rotation around vertical axis and temperature control of the sample over the range of 20-700 K. A gas handling system was also used for the introduction of $\mathrm{Ar}$ or $\mathrm{CH}_{4}$. 


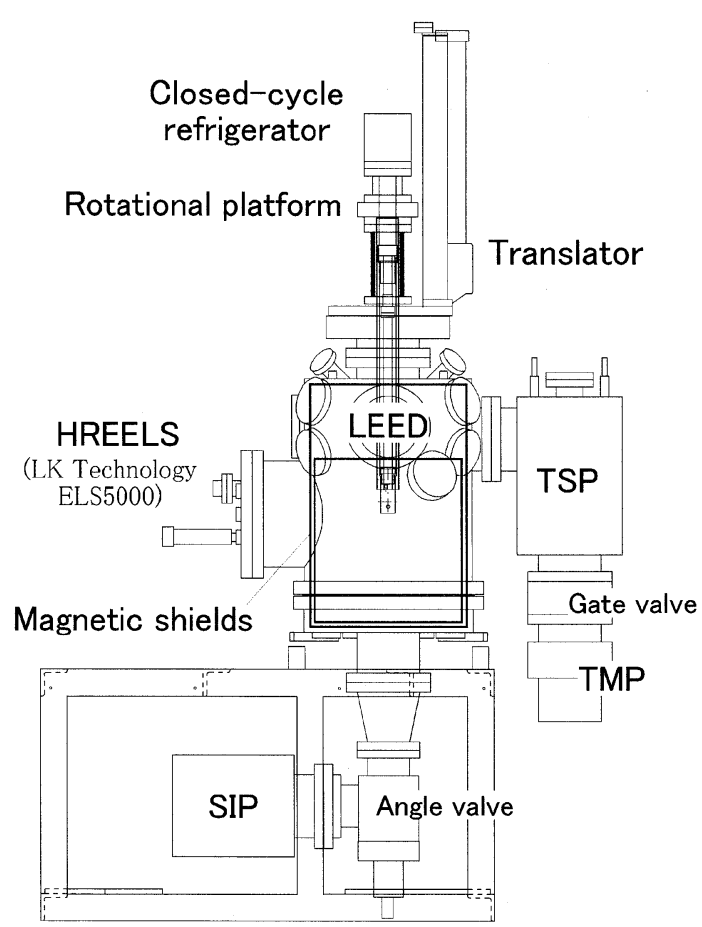

Fig. 1 Schematic diagram of the experimental apparatus.

The ultimate pressure less than $1 \times 10^{-8} \mathrm{~Pa}$ was obtained after bakeout procedure for 24-48 hours. The x-ray-aligned and chemically polished $\mathrm{Ag}(111)$ substrate was cleaned by standard procedures involving low-energy Ar-ion bombardment and heating cycles, which was repeated until clear diffraction spots could be observed. The layer of $\mathrm{CH}_{4}$ physisorbed on the $\mathrm{Ag}(111)$ substrate was formed under adsorption equilibrium conditions at the substrate temperature ranging from 20 to $50 \mathrm{~K}$. The LEED patterns of $\mathrm{CH}_{4}$ monolayer were recorded by a liquid $\mathrm{N}_{2}$ cooled $\mathrm{CCD}$ camera (Princeton Instruments). In the course of the experiments, it has turned out that the adsorbed layer is very easy to be disturbed by the incident electron beam. Then to minimize the irradiation effect, the electron source of LEED is activated to emit the electron current of less than $1 \mathrm{nA}$ during only the exposure.

\section{Results and Discussion}

The typical LEED patterns of $\mathrm{CH}_{4}$ monolayer are shown in Fig. 2. They were recorded at the incident electron exposure of $3 \mathrm{nC}$, which is necessary to obtain LEED patterns with good contrast. The incident energy is $56 \mathrm{eV}$. Fig. 2(a) was obtained with the $\mathrm{CH}_{4}$ pressure of $1 \times 10^{-6} \mathrm{~Pa}$ and the sub-

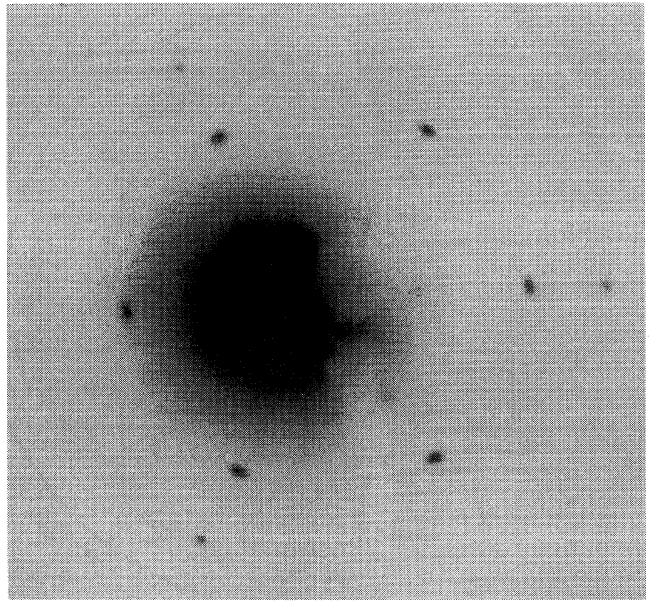

(a)

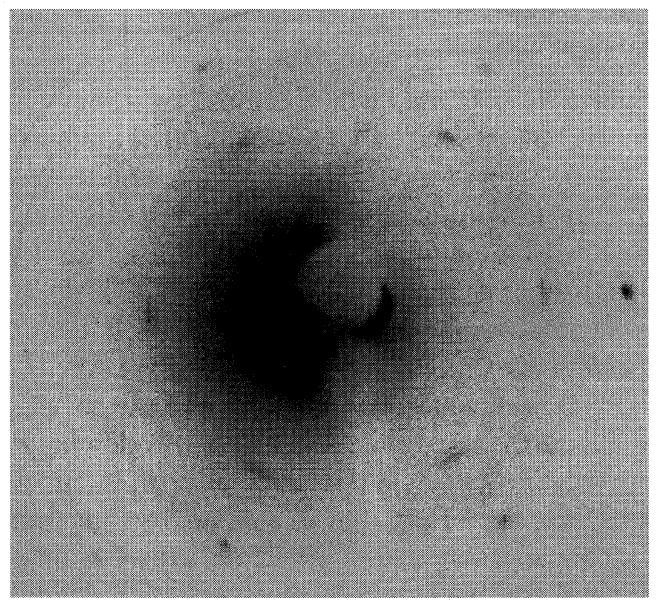

(b)

Fig. 2 LEED patterns of physisorbed $\mathrm{CH}_{4}$ on $\mathrm{Ag}(111)$. (a) was obtained with the $\mathrm{CH}_{4}$ pressure of $1 \times 10^{-6} \mathrm{~Pa}$ and the substrate temperature of $37 \mathrm{~K}$. (b) was obtained with the $\mathrm{CH}_{4}$ pressure of $5 \times 10^{-6}$ $\mathrm{Pa}$ and the substrate temperature of $43 \mathrm{~K}$.

strate temperature of $37 \mathrm{~K}$ and Fig. 2(b) was obtained with the $\mathrm{CH}_{4}$ pressure of $5 \times 10^{-6} \mathrm{~Pa}$ and the substrate temperature of $43 \mathrm{~K}$. Hexagonal spots with similar intensity observable in both figures correspond to the crystal of $\mathrm{CH}_{4}$. The patterns indicate that $\mathrm{CH}_{4}$ crystal forms rotationally ordered incommensurate phase similar to $\mathrm{Xe}$ monolayer formed on graphite and $\mathrm{Ag}(111)$ substrates. These $\mathrm{CH}_{4}$ spots could be observed over the incident electron energy range of 30 to $100 \mathrm{eV}$. The lattice constant of $\mathrm{CH}_{4}$ monolayer derived from the LEED 


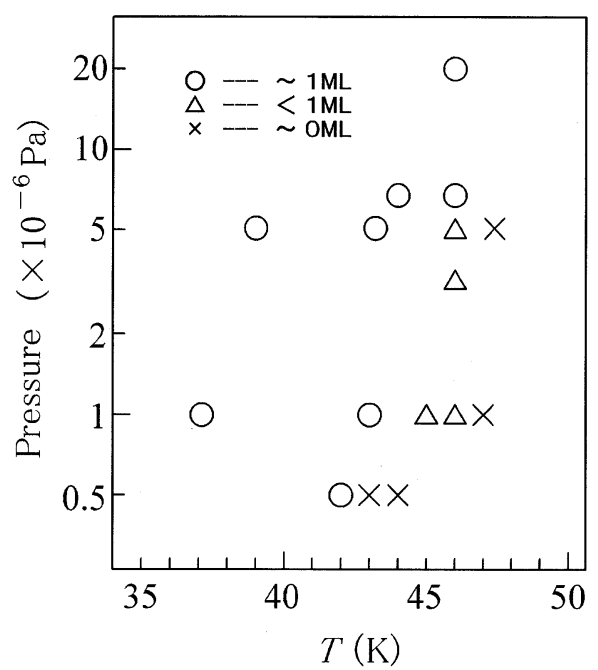

Fig. 3 Adsorption condition dependence of coverage derived from the LEED intensity.

patterns is $0.41 \mathrm{~nm}$, which almost coincides with that of bulk crystal of $\mathrm{CH}_{4}(0.42 \mathrm{~nm})$. Though the diffraction patterns are almost same for the two pictures, the ratios of spot intensities of $\mathrm{CH}_{4}$ peaks to $\mathrm{Ag}$ ones are different, which means that the $\mathrm{CH}_{4}$ coverage in Fig. 2(a) is higher than in Fig. 2(b). The absolute coverage can not be estimated directly from the spot intensity, however, the coverage for Fig. 2(a) is considered to be unity and the coverage for Fig. 2(b) is less than unity due to following experimental results; (1) the ratios of peak intensities of $\mathrm{CH}_{4}$ to $\mathrm{Ag}$ appeared to be similar for wide range of adsorption conditions, i.e. ambient $\mathrm{CH}_{4}$ pressure and substrate temperature, and (2) if the substrate is cooled further at the constant ambient pressure in $10^{-7} \sim 10^{-6} \mathrm{~Pa}$ range, the spots of both $\mathrm{CH}_{4}$ and $\mathrm{Ag}$ diminishes together while the background intensity goes higher. This means only the first $\mathrm{CH}_{4}$ layer easily forms single-crystal structure, on the other hand, the succeeding layers become disordered one.

As a result of the observation of LEED patterns indicating the formation of $\mathrm{CH}_{4}$ crystal over comprehensive adsorption conditions, the ratio of spot intensities of $\mathrm{CH}_{4}$ to $\mathrm{Ag}$ is roughly classified into two categories; typically Fig. 2(a) (type I) and Fig. 2(b) (type II). In Fig. 3, the adsorption conditions showing LEED pattern of each type are indicated as $\bigcirc$ for type I, and $\triangle$ for type II. The adsorption conditions showing no $\mathrm{CH}_{4}$ spots are indicated with $x$. We did not try to determine the boundary between monolayer and multilayer phases. Fig. 3 exhibits the presence of intermediate phase where less than monolayer of $\mathrm{CH}_{4}$ molecules adsorb in incommensurate lattice on $\mathrm{Ag}(111)$ substrate in equilibrium with gas phase. This is not the case for the adsorption of rare gas on graphite or metal substrates; rare gasses adsorb in commensurate lattice $(\sqrt{2}$ structure) before the coverage saturates at monolayer. The lattice constants are almost the same for both type I and II, however, more careful analysis leads to the finding that the lattice spacing for type II is $1-2 \%$ larger than that of type I. Moreover the intensity profile of $\mathrm{CH}_{4}$ spots for type II is expanded toward azimuthal direction. This indicates that the lattice direction of crystalline islands tends to have narrow distribution under unsaturated coverage while the lattice spacing remains at a specific value. The slight difference in the lattice constant between monolayer and bulk crystal may cause this translation and deformation of $\mathrm{CH}_{4}$ spots.

Low energy I-V curves of specular diffraction for $\mathrm{CH}_{4} / \mathrm{Ag}(111)$ system have been measured before using HREELS. ${ }^{11)}$ The I-V curve is modulated by diffraction effect, and there is ambient-pressure dependence in the modulation of $\mathrm{I}-\mathrm{V}$ curves. ${ }^{12)}$ The data were measured at the $\mathrm{CH}_{4}$ pressure of $3 \times 10^{-8}$ and $1 \times 10^{-7} \mathrm{~Pa}$ with the substrate temperature of $43 \mathrm{~K}$. Though the pressure range is lower than in Fig. 3, it could be argued that the phase boundary between monolayer and submonolayer regions in Fig. 3 is extrapolated to the adsorption condition between $3 \times 10^{-8}$ and $1 \times 10^{-7} \mathrm{~Pa}$ with the substrate temperature of $43 \mathrm{~K}$, then the ambient-pressure dependence in the modulation of $\mathrm{I}-\mathrm{V}$ curve seen in ref.12 arises from the difference of $\mathrm{CH}_{4}$ coverage.

The difficulty of the LEED observation of physisorbed $\mathrm{CH}_{4}$ layer is high probability of electron stimulated desorption. If we continued to observe the LEED image at the adsorption condition of type I, the intensity and profile of $\mathrm{CH}_{4}$ spots changed towards those of type II along with the irradiation of electron beam. The $\mathrm{CH}_{4}$ spots disappeared after the irradiation of $1 \mu \mathrm{C}$ at the incident energy of $56 \mathrm{eV}$. If we assume the size of electron beam in the range of 0.3 to $1 \mathrm{~mm}$ on the sample, the desorption yield is estimated at $0.1-1$.

\section{Summary}

LEED patterns of $\mathrm{CH}_{4}$ monolayer physisorbed on $\mathrm{Ag}(111)$ were observed at various adsorption equilibrium conditions. Diffraction spots of $\mathrm{CH}_{4}$ monolayer crystal were clearly observed at $5 \times 10^{-7}$ $\sim 2 \times 10^{-5} \mathrm{~Pa}$, substrate temperature around $40 \mathrm{~K}$. The diffraction pattern indicates $\mathrm{CH}_{4}$ crystal forms rotationally ordered incommensurate phase similar 
to rare gas crystals. Under specific equilibrium condition, intermediate state with the coverage less than unity exists, which is a different feature than those of rare gas layers.

\section{References}

1) H. Ohtani, C.-T. Kao, M. A. Van Hove and G. A. Somorjai: Prog. Surface Sci., 23 (1986) 155.

2) H. You, S. C. Fain, Jr., S. Satija and L. Passell: Phys. Rev. Lett., 56 (1986) 244.

3) J. Cui and S. C. Fain Jr.: J. Vac. Sci. Technol., A5 (1987) 710 .

4) M. Sakurai, T. Okano and Y. Tuzi: J. Vac. Soc. Jpn., 29 (1985) 451 [in Japanese].

5) S. Igarashi, Y. Abe, T. Hirayama and I. Arakawa:
J. Vac. Sci. Tech., A16, (1998) 974-8.

6) J. E. Demuth, D. Schmeisser and P. Avouris: Phys. Rev. Lett., 47 (1981) 1166.

7) L. Sanche and M. Michaud: Phys. Rev., B30 (1984) 6078.

8) M. Sakurai, T. Okano and Y. Tuzi: J. Vac. Sci. Technol., A5 (1987) 431-434.

9) M. Sakurai, T. Okano and Y. Tuzi: Japn. J. Appl. Phys., 26 (1987) L1651-L1653.

10) R. E. Palmer and P. J. Rous] Rev. Mod. Phys., 64 (1992)383.

11) M. Sakurai, T. Okano and Y: Tuzi, Vacuum, 41 (1990) 234-236.

12) M. Sakurai: Thesis (University of Tokyo, 1988) [in Japanese]. 